\title{
How to Rapidly Convert General Wards to ICUs during the COVID-19 Epidemic: Experience from Wuhan, Chin
}

\author{
Mei He${ }^{1}$, Jie Xiong ${ }^{1}$, Luhong $\mathrm{Hu}^{1}$, Sufang Huang ${ }^{1}$, Zhaoxia $\mathrm{Li}^{1}$, Chunling Guo ${ }^{1}$, and Juan \\ Deng ${ }^{1}$
}

${ }^{1}$ Tongji Hospital of Tongji Medical College of Huazhong University of Science and Technology

April 28, 2020

\begin{abstract}
Background : Critically ill patients with coronavirus disease 2019(COVID-19)was surging and far outnumbered existing beds. Aims : To describe how to rapidly convert general wards to intensive care units for critically ill patients with COVID19. Materials \& Methods : Comprehensive assessment and analysis of available resources and standard requirements. Results : The ICUs were successfully assembled in four days. The conversion included environment reconstruction, configuration and management of equipment, information system construction and human resource allocation. A total of 172 critically ill patients had been admitted to the contemporary ICUs and none medical staff was infected. Discussion : The epidemic situation of COVID-19 poses a great challenge to various management departments of the hospital, especially for critically ill patients with high mortality rate. To save more critically ill patients, the conversion of a general ward to a quarantine ICU ward must be completed in a short time, and the optimal allocation of resources must be appropriate to ensure that the medical team works effectively and is of high quality. In face of the overloaded medical system, the ideal non-negative pressure ward is hard to achieve. However, we have demonstrated with evidence that our conversions are effective in both providing care to the critical patients and protecting the safety of our staff. Conclusion: The conversion is successful and the running experience would be a reference for hospitals in other areas nationally or globally.
\end{abstract}

Since January 2020, many cases of coronavirus disease 2019 have been confirmed in China. Local officials had reported 47,647 new cases of COVID-19 as of 24:00 on 21/2/2020, of which 10,892 were severe cases (National Health Commission of the People's Republic of China, 2020). The mortality rate of critically ill patients reached over 50\% (World Health Organization, 2020a). To improve the treatment capacity for critically ill patients and reduce mortality, one site at our hospital was opened to admit patients with COVID-19. The original intensive care unit at this site only had 20 beds. However, due to the fact that critically ill patients far outnumbered existing beds, and the fact that the use of the 10,000-level laminar flow system in the existing ICU was not conducive to protecting medical staff, decision was made to convert two general wards into two temporary ICUs, adding 72 beds for the critical ill. To date, the two temporary ICUs run well. The experience of assembling ICUs is described as follows.

\section{Ward Environment Reconstruction}

\subsection{Standard infectious ward layout}

Originally, there were two wards on each floor, located in the East area and West area with a corridor between them. Thus, the doors in the elevator area were blocked to connect the East and West areas as a whole. Two additional doors were added to the original staff passage in the West area and the original patient corridor in the East area to form two buffer zones, forming a polluted area - buffer room - semi-polluted area - buffer room - cleaning area layout (from west to east). The West district served as a contaminated area ward 
for critically ill patients with COVID and for removing the outermost protective equipment. The section between West ward and East ward formed a semi-polluted area, where medical care workstations, dispensing rooms, warehouses, and staff toilets were set up. Staff also took off inner clothing here. The East ward was set up as the staff-only clean areas, which are used by staff to don protective equipment and store materials and as staff living areas (Figure 1). The old staff passageway in the East district was retained as the staff passageway, and the original dirt elevator, located in the west ward, was converted into a patient passageway. The original patient access door was blocked, and a continuously running air curtain was installed near the ceiling above the door to promote the air to flow from the clean area to the polluted area.

\subsection{Ward settings}

Hospital infection prevention and control standards for the intensive care unit require that each ICU bed area should be no less than $15 \mathrm{~m}^{2}$ and that the bed spacing should be greater than one meter (National Health Commission of the People's Republic of China, 2016). The original two general wards had 36 rooms, all of which were triple rooms with below-standard bed spacing. Therefore, the triple rooms were transformed into double rooms, which greatly increased the bed spacing to more than one meter. For infectious wards, negative airflow rooms are ideal. However, temporarily established ICUs were exempted from meeting this criterion so long as the ward was well-ventilated. Therefore, each ward was equipped with two exhaust fans on the windows (approximately one meter above the ground) for continuous ventilation, and the ventilation provided by each fan was ensured to be at least $160 \mathrm{~L} / \mathrm{s}$ (World Health Organization, 2020b). We opened the doors and windows of the consultation room and temporary storage room directly behind the nurse working station so that the air flowed from the clean area to the polluted area and was finally discharged out of the window. Except for the ceiling of the contaminated ward in the West district, the ceilings of the other areas were all sealed with nonwoven fabrics to avoid collusion with the air. To meet the needs of large numbers of critical patients using oxygen at the same time, we increased the pressure of the central oxygen supply equipment in the ward. .

\section{Configuration and Management of Equipment}

\subsection{Equipment selection and check}

Medical equipment manufacturers had donated many products with different bands, including blood gas analyzers, continuous renal replacement therapy (CRRT) instruments and extracorporeal membrane oxygenation machines (ECMO). Personnel were required to participate in equipment training, albeit efforts were made to coordinate medical teams from different hospitals to use brands and models that they were familiar with to minimize training time. At the same time, it was necessary to implement a reserve of special consumables matching the instrument.

\subsection{Equipment management}

To ensure that rescue equipment was efficiently accessible to the entire ward, each ICU ward was equipped with two emergency vehicles, with one placed in the front ward and the other in the back ward. Two defibrillators were stored in the nurse working station. Each bed was equipped with an independent ECG monitor, a cart and a set of disinfection supplies. To minimize cross-infection, we stopped the original central suction equipment, and each bed was equipped with an electric negative pressure suction device. Each ward was equipped with 20 high-flow airway humidification devices, 30 noninvasive ventilators and 30 invasive ventilators with air compressors. In addition, it was also equipped with two fiberoptic bronchoscopes, two electrocardiographs, two vibration expectorators, two blood gas analyzers and one portable bedside ultrasounds. Nonconventional ICU equipment such as blood purification machines, ECMO machines and packages were placed in storage areas in the semi-contaminated areas. In emergency situations, this equipment was easily accessible and not contaminated. We placed more than 40 medium rectangular storage boxes at the nurses' workstation to store common disposable consumables by their functionality, such as "airway consumables", and "venous therapy use". Eye-catching labels were used for the convenience of medical personnel wearing goggles and face shields. 


\section{Information System Construction}

Each ward was equipped with a central real-time monitoring system that displayed the vital signs of all patients on the monitoring screens in the nurses' workstation and the medical station in the semi-contaminated area. In each of the three zones, physician information stations and nurse information stations were equipped with four computers. They were used for document writing and prescribing medical orders. A certain number of walkie-talkies were provided to each of the three areas for calls and communication between staff. Each of the three districts was equipped with a smart phone that could save and transmit documents, recording and images. Additionally, we authorized all medical staff, including out-of-province medical staff who came to support Wuhan, to use open authority management, and reformat various document templates and doctor order templates to ensure consistency.

\section{Human Resource Allocation}

\subsection{Establishment of a multidisciplinary team}

After the epidemic outbreak, medical staff from all over the country came to provide aid. The two quarantine ICUs uniformly set up positions and determined job responsibilities. Here we present the human resource allocation strategy for one unit. Firstly, a multidisciplinary team was formed, comprising of 25 physicians, 138 nurses, 24 nurse assistants, six personals from infection control departments, two sonologists, one dietician, one rehabilitation therapist, one psychological therapist and one radiologist. Additionally, there were four experts took turns to make the rounds of the wards.

\section{2 nurse staff allocation and support}

According to the requirements of Nosocomial Infection Prevention and Control ${ }^{[3]}$, the ICU should be equipped with a sufficient number of professional medical staff. At that time, there were 138 nursing staff, including 135 nurses and 3 head nurses, in charge of 36 beds. The ratio of the number of nurses to the actual number of beds was 3.8:1. We adopted a 4 -hour shift model. That is, they worked 4 hours a day for 6 consecutive days and took a day off after six working days. The psychological counseling hotline in the mental hospital was open 24 hours a day. Staff could call it for free whenever they need to.

There are 18 primary nurses on duty during each shift. On average, one nurse is in charge of two critically ill patients. Two additional nurses are specialized in dealing with medical orders, informing corresponding primary nurses, admitting patients, documenting critical values from the clinical laboratory, replenishing supplies and rescue drugs, checking accounts and discharging patients. One nurse is responsible for the preparation of medicines for all patients in the ward and the replenishment of commonly used medicine. The persons from the infection control department hold the supervising position, whose job responsibility includes checking and ensuring that all personnel wear protective equipment appropriately when entering the ward, supplying consumable items and clearing the semi-polluted area. One addtional position was for staff catering, transportation and accommodation.

We set a lead nurse on each shift, who had the responsibilities of managing, inspecting, supervising, guiding, and assigning patients to each primary nurse. Each team consisted of nurses with ICU backgrounds and non-ICU backgrounds. The combination of different working years was another consideration.

\section{Running Effect}

From 4/2/2020 to3/4/2020, the wards have been running for 59 days. A total of 172 critically ill patients with COVID-19 were admitted to the temporary ICUs. Oxygen therapy is a key component of treatment for patients with COVID-19 and mechanical ventilation was commonly used in the critical ill. We had 126 patients who received invasive mechanical ventilation and 68 patients who received noninvasive ventilation during hospitalization. Fiberoptic bronchoscopy examination, as a high-risk procedure, was also performed 43 times for diagnosis and treatment. To protect the involved staff in this procedure, we developed a revised standards for this procedure, including detailed preparation prior to exam, precaution strategies and decontamination of bronchoscope and environment. ECMO therapy is the last resort for maintaining 
critical patients' respiratory and cardiac function. Six patients had undergone this treatment in our unit. Additionally, 281 CRRTs and 15 tracheotomies were successfully carried out at the patients' bedsides. In spite of the heavy workload and high-risk working environment, none of the staff were infected or had any mental health problems, fortunately.

Acknowledgment: The work is supported by the Nature Science Foundation of Hubei Province [No.2019CFB645] for language editing.

\section{Conflicts of interest : None.}

\section{References}

National Health Commission of the People's Republic of China, 2016. Hospital Infection Prevention and Control Standards for the Intensive Care Unit [WS/T509].http://www.nhc.gov.cn/wjw/s9496/201701/1f9de66563304061a4fcd7f54 20.03.26).

National Health Commission of the People's Republic of China, 2020. Update on COVID-19 outbreak.http://www.nhc.gov.cn/ 20.02.21).

World Health Organization. 2020a. Coronavirus disease 2019 (COVID-19)

Situation Report-41. https://www.who.int/docs/

default-source/coronaviruse/situation-reports/20200301-sitrep-41-covid-19.

pdf?sfvrsn=6768306d_2(accessed 20.04.16).

World Health Organization, 2020b. Infection prevention and control during health care when novel coronavirus (nCoV) infection is suspected, interim guidance.https://www.who.int/internal-publications-detail/surveillancecase-definitions-for-human-infection-withnovel-coronavirus-(ncov)(accessed 20.03.20).

\section{Hosted file}

Figure 1. doc available at https://authorea.com/users/314131/articles/444518-how-to-rapidly-convertgeneral-wards-to-icus-during-the-covid-19-epidemic-experience-from-wuhan-chin 